\title{
MicroRNA-27b inhibits the development of melanoma by targeting MYC
}

\author{
YI TIAN $^{1}$, JUANNI ZENG ${ }^{2}$ and ZONGLIANG YANG ${ }^{2}$ \\ Departments of ${ }^{1}$ Dermatology and ${ }^{2}$ Anorectal Disease, The Second Affiliated Hospital, \\ Hunan University of Chinese Medicine, Changsha, Hunan 410005, P.R. China
}

Received June 14, 2020; Accepted February 2, 2021

DOI: 10.3892/ol.2021.12631

\begin{abstract}
Cutaneous malignant melanoma is a malignancy with one of the fastest increasing incidence rates worldwide; however, the mechanism underlying the occurrence and development of melanoma remains unclear. The aim of the present study was to identify novel biomarkers for the occurrence and development of melanoma. The results of the present study demonstrated that the expression levels of microRNA (miR)-27b were decreased in melanoma tissue samples compared with those in adjacent noncancerous tissue samples and cells according to online and experimental data. By contrast, MYC expression levels were upregulated in melanoma compared with those in adjacent noncancerous tissue samples. miR-27b overexpression significantly inhibited A375 and A2085 melanoma cell DNA synthesis, viability and invasive ability. Dual-luciferase reporter assay results demonstrated that miR-27b inhibited MYC expression through binding to the 3'-untranslated region of MYC mRNA. MYC knockdown in melanoma cells exerted similar effects to those of miR-27b overexpression on DNA synthesis, cell viability and invasive ability; the effects of miR-27b inhibition were significantly reversed by MYC knockdown. In conclusion, the miR-27b/MYC axis may modulate malignant melanoma cell biological behaviors and may be a potential target for melanoma treatment.
\end{abstract}

\section{Introduction}

Cutaneous malignant melanoma is a malignancy with one of the fastest increasing incidence rates worldwide $(1,2)$. Although melanoma accounts for only a limited proportion of all skin malignancies, it causes the largest number of skin

Correspondence to: Dr Zongliang Yang, Department of Anorectal Disease, The Second Affiliated Hospital, Hunan University of Chinese Medicine, 233 Caie North Road, Changsha, Hunan 410005, P.R. China

E-mail: 1988yzlmt@sina.cn

Key words: melanoma, microRNA-27b, MYC, proliferation, invasion cancer-related deaths, with $\sim 55,500$ deaths each year (3). The high mortality of melanoma mainly occurs due to distant metastasis (4). Immunotherapy has transformed the treatment of melanoma in the past decade, with a significant increase in overall survival (5). For example, an antibody against cytotoxic T-lymphocyte-associated protein 4 (CTLA-4), ipilimumab, increased the 2-year survival rates of patients with metastatic melanoma from 14 to $24 \%$ (6). With the application of monoclonal antibodies against inhibitory immune checkpoints, such as CTLA-4 and anti-programmed death 1, the treatment efficacy for metastatic melanoma has improved $(5,7)$. However, predictive biomarkers for melanoma are still lacking. The occurrence and progression of melanoma is considered to result from disorders of the oncogenic and tumor inhibitory pathway functions (8). The importance and relevance of various biomarkers have been demonstrated in melanoma studies $(9,10)$, and targeted agents such as vemurafenib and dabrafenib have enhanced the survival of patients with melanoma; however, patient prognosis remains poor (3). Therefore, it is crucial to study novel biomarkers that drive the occurrence and development of melanoma, which may contribute to the development of new diagnostic and treatment targets.

The Wnt/ $\beta$-catenin pathway contributes to cancer metastasis through regulating the epithelial-mesenchymal transition (EMT) $(11,12)$, which has been reported in melanoma (13). The $\mathrm{Wnt} / \beta$-catenin signaling pathway is often abnormally activated and may participate in the occurrence and development of tumors (14), leading to $\beta$-catenin nuclear translocation and the transcription of downstream target genes, such as MYC (15). The MYC gene is typically expressed constitutively in malignancies, and c-Myc, which is encoded by MYC, has been reported to regulate the gene expression of $\leq 15 \%$ of the human genome (16). From a clinical perspective, high expression levels of c-Myc protein exhibit a significant association with distant metastasis and impaired prognosis; from a biological perspective, the overexpression of c-Myc results in a significant increase in cell viability, invasion and migration (17). Furthermore, c-Myc increases the protein expression levels of Snail, which is an EMT marker, in vivo and in vitro (18). Thus, MYC may be a novel therapeutic target in melanoma, and the suppression of MYC expression may be an effective therapeutic option for patients with melanoma.

MicroRNAs (miRNAs), a type of noncoding RNAs that are $<22$ nucleotides long, bind to the 3 -untranslated region 
(3'-UTR) of their downstream target mRNAs, inhibiting their expression $(19,20)$. Depending on the extent of base pairing, miRNAs lead to translational repression, mRNA deadenylation or decay (21). Changes in miRNA expression levels contribute to human tumor occurrence and development (19). In melanoma, deregulation of miRNA expression levels has also been reported previously. Using C57BL/6 mice, Noori et al (22) have demonstrated that high levels of miRNA (miR)-30a expression in melanoma suppress metastasis in vivo through binding to zinc finger E-box-binding homeobox 2 and E-cadherin. A series of miRNAs, such as miR-1908, miR-199a-5p and miR-199a-3p, have been reported as endogenous factors promoting melanoma metastatic invasion, angiogenesis and colonization (23). Since distinct miRNA expression profiles have been investigated at various stages of melanoma progression $(24,25)$, identifying additional miRNAs that target MYC may provide new therapeutic options for melanoma treatment.

The aim of the present study was to identify a miRNA/mRNA axis that may modulate the biological malignant behaviors of melanoma cells in vitro. To achieve this, an online tool was used to select the candidate miRNAs that could bind to the MYC 3'UTR, and the effects of the miR-27b/MYC axis on melanoma progression were determined.

\section{Materials and methods}

Clinical sampling. A total of 18 paired melanoma and adjacent noncancerous tissue samples ( $5 \mathrm{~cm}$ from the edge of the tumor) were collected from patients who received surgical treatment for cutaneous melanoma at the Second Affiliated Hospital of Hunan University of Chinese Medicine (Changsha, China) with the approval of the Ethics Committee of the Second Affiliated Hospital of Hunan University of Chinese Medicine (approval no. 2019-KY-031). All patients provided written informed consent. The obtained clinical samples were stored at $-80^{\circ} \mathrm{C}$ or fixed in $10 \%$ formalin at room temperature until further use.

Hematoxylin and eosin (H\&E) and immunohistochemical (IHC) staining. The histomorphological changes in melanoma and adjacent noncancerous tissues were evaluated by $\mathrm{H} \& \mathrm{E}$ staining at room temperature. The $5-\mu \mathrm{m}$ sections were stained with hematoxylin solution for $5 \mathrm{~min}$, destained with $0.5 \%$ acid ethanol ( $\mathrm{pH} 2.0)$ and stained with eosin for $30 \mathrm{sec}$. Following dehydration with graded ethanol $(80,90,95 \%$ and absolute ethanol incubated for 3 min each) and clearing with xylene, the sections were mounted with neutral balsam and observed under an optical microscope (Olympus Corporation) with x100 magnification in three fields per sample.

The protein content and distribution of c-Myc in tissue samples were detected by IHC as previously described (26). Briefly, $5-\mu \mathrm{m}$ tissue sections were deparaffinized in xylene and rehydrated using graded ethanol (absolute ethanol, 95, 90, 80 and $75 \%$ ethanol incubated for 3 min each) in PBS. Endogenous peroxidase was blocked with $0.3 \%$ hydrogen peroxide for $10 \mathrm{~min}$ at room temperature. The sections were incubated with $5 \%$ normal rat serum (Beijing Solarbio Science \& Technology Co., Ltd.) at room temperature for $10 \mathrm{~min}$, followed by incubation with anti-c-Myc (1:200, cat. no. ab32072; Abcam) at $4^{\circ} \mathrm{C}$ overnight. Subsequently, the sections were incubated with an HRP-polymer-conjugated anti-rabbit/mouse secondary antibody (1:500; cat. no. SV0004; Wuhan Boster Biological Technology, Ltd) at $37^{\circ} \mathrm{C}$ for $30 \mathrm{~min}$ and stained using a diaminobenzidine staining kit (Wuhan Boster Biological Technology, Ltd.). The nuclei were counterstained with hematoxylin. The sections were mounted with neutral balsam and observed under an optical microscope (Olympus Corporation) with x100 magnification in three fields per sample.

Bioinformatics analysis. For miRNA target gene prediction, the online tool TargetScan was used (http://www. targetscan.org/vert_72/) (27). To analyze the differentially expressed miRNAs $(\mid \log \mathrm{FCl}>0.56 ; \mathrm{P}<0.05)$ in melanoma cells, the GEO dataset GSE77090 (https://www.ncbi.nlm.nih. gov/geo/query/acc.cgi?acc=GSE77090) was downloaded and analyzed using the 'Limma' package in R $(28,29)$.

Cell lines and culture. Normal human melanocytes (MC) were obtained from ATCC (cat. no. PCS-200-013) and cultured using an Adult Melanocyte Growth kit (cat. no. PCS-200-042; ATCC). The human melanoma cell lines A375 and A2058 were obtained from ATCC (cat. nos. CRL-1619 and CRL-11147) and cultured in DMEM (cat. no. 30-2002) supplemented with $10 \%$ fetal bovine serum (FBS; Invitrogen; Thermo Fisher Scientific, Inc.), $100 \mathrm{mg} / \mathrm{m}$ penicillin and $100 \mathrm{U} / \mathrm{ml}$ streptomycin. The cells were incubated with $5 \% \mathrm{CO}_{2}$ at $37^{\circ} \mathrm{C}$.

Cell transfection. For miR-27b overexpression or inhibition, miR-27b mimics, mimics-negative control (NC), miR-27b inhibitor or inhibitor-NC were transfected into A375 and A2058 cells $\left(1 \times 10^{6}\right.$ cells $\left./ \mathrm{ml}\right)$. MYC knockdown was achieved by transfection of small interfering (si)RNA specific to MYC (si-MYC). All transfection vectors (final concentration, $20 \mathrm{nM}$ ) were synthesized by Shanghai GenePharm Co., Ltd. The transfections were performed using Lipofectamine ${ }^{\circledR} 3000$ (Invitrogen; Thermo Fisher Scientific, Inc.) at $37^{\circ} \mathrm{C}$ for $6 \mathrm{~h}$. Subsequently, the culture medium was replaced with fresh culture medium. At $48 \mathrm{~h}$ post-transfection, the cells were harvested for further experiments. The sequences of the miR-27b mimics, inhibitor, si-MYC and the corresponding NC vectors are listed in Table I.

5-bromo-2-deoxyuridine (BrdU) assay. The DNA synthesis ability of the cells was examined using BrdU assay by determining the BrdU incorporation by proliferating cells. A375 and A2058 cells were transfected with the miR-27b mimics, inhibitor or si-MYC for $48 \mathrm{~h}$. Subsequently, $1 \times 10^{4}$ A 375 and A2058 cells were cultured in 24-well plates for $8 \mathrm{~h}$ and incubated with $10 \mu \mathrm{g} / \mathrm{ml} \mathrm{BrdU}$ (Sigma-Aldrich; Merck KGaA) for 24 and $48 \mathrm{~h}$ at $37^{\circ} \mathrm{C}$ with $5 \% \mathrm{CO}_{2}$; subsequently, the BrdU solution was removed. Following fixation by $4 \%$ paraformaldehyde and permeabilized by $0.1 \%$ Triton for $10 \mathrm{~min}$ each at room temperature, the cells were incubated sequentially with a BrdU antibody (1:50; cat. no. 5290; Cell Signaling Technology, Inc.) at $4^{\circ} \mathrm{C}$ overnight and an HRP-goat anti-mouse secondary antibody (1:200; cat. no. A0216; Beyotime Institute of Biotechnology) for $1 \mathrm{~h}$ at room temperature. The optical density at $450 \mathrm{~nm}$ was measured using a microplate reader (Bio-Rad Laboratories, Inc.).

MTT assay. The MTT assay was used to determine the cell viability. A375 and A2058 cells transfected with the miR-27b 
Table I. Primer and microRNA sequences.

A, Primers used for RT-quantitative PCR

\begin{tabular}{|c|c|}
\hline Gene & Sequences $\left(5^{\prime} \rightarrow 3^{\prime}\right)$ \\
\hline \multirow[t]{3}{*}{$\operatorname{miR}-27 b-5 p$} & RT: GTCGTATCCAGTGCGTGTCGTGGAGTCGGCAATTGCACTGGATACGACGTTCAC \\
\hline & F: GCCAGAGCTTAGCTGATTG \\
\hline & R: CAGTGCGTGTCGTGGA \\
\hline \multirow[t]{2}{*}{ MYC } & F: GGCTCCTGGCAAAAGGTCA \\
\hline & R: CTGCGTAGTTGTGCTGATGT \\
\hline \multirow[t]{2}{*}{ GAPDH } & F: ACAGCCTCAAGATCATCAGC \\
\hline & R: GGTCATGAGTCCTTCCACGAT \\
\hline \multirow[t]{2}{*}{ U6 } & F: CTCGCTTCGGCAGCACA \\
\hline & R: AACGCTTCACGAATTTGCGT \\
\hline \multirow[t]{2}{*}{ MYC (RIP assay) } & F: GCCTTGGTTCATCTGGGTCTAA \\
\hline & R: TGGGGTTGATGTAGAGTTAGGGAT \\
\hline
\end{tabular}

B, Vectors used for transfections

\begin{tabular}{ll}
\hline Oligomer & \multicolumn{1}{c}{ Sequences $\left(5^{\prime} \rightarrow 3^{\prime}\right)$} \\
\hline Mimics NC & S: UUCUCCGAACGUGUCACGUTT \\
miR-27b mimics & AS: ACGUGACACGUUCGGAGAATT \\
& S: AGAGCUUAGCUGAUUGGUGAAC \\
Inhibitor NC & S: UCACCAAUCAGCUAAGCUCUUU \\
miR-27b inhibitor & S: GUUCACCAAUCAGCUAAGCUCU \\
Wt-MYC 3'-UTR & S: aattctaggcgatcgctcgagAGATAATACAAAGCAGCAATCTGGAC \\
Mut-MYC 3'-UTR & AS: atttattgcggccagcggccgcTTCCCTATCAGTGAATCTTGGGC \\
Si-NC & CACAAATAGAGtaggcCATTGTTATGACTTGAGTCTGTCCATT \\
Si-MYC & S: UUCUCCGAACGUGUCACGUTT \\
& AS: ACGUGACACGUUCGGAGAATT \\
& S: AGAAUGAUUAAAAUAACCCTT \\
& AS: GGGUUAUUUUAAUCAUUCUTT \\
\hline
\end{tabular}

RT, reverse transcription; miR, microRNA; RIP, radioimmunoprecipitation; F, forward; R, reverse; NC, negative control; Wt, wild-type; UTR, untranslated region; Mut, mutant; si, small interfering RNA; S, sense; AS, antisense.

mimics, inhibitor or si-MYC were collected and, following 24-h culture in 96 -well plates $\left(5 \times 10^{3}\right.$ cells/well $)$ at $37^{\circ} \mathrm{C}$, the cells were supplemented with $20 \mu \mathrm{l}$ MTT (5 mg/ml; Sigma-Aldrich; Merck $\mathrm{KGaA}$ ) and incubated for $4 \mathrm{~h}$ in a humidified incubator at $37^{\circ} \mathrm{C}$. Subsequently, the supernatant was removed, and $200 \mu \mathrm{l}$ DMSO was added to dissolve the formazan crystals. The optical density at $490 \mathrm{~nm}$ was measured using a microplate reader.

Transwell assay. The invasive ability of A375 and A2058 cells was determined using a Transwell invasion assay. A375 and A2058 cells transfected with the miR-27b mimics, inhibitor or si-MYC were collected, suspended in FBS-free DMEM and seeded into the upper chambers of the Transwell inserts pre-coated with Matrigel for $4 \mathrm{~h}$ at $37^{\circ} \mathrm{C}$. DMEM with $10 \%$ FBS was added to the lower chambers. Following 24-h culture at $37^{\circ} \mathrm{C}$, cells adhering to the upper surface of the membrane were removed with a cotton swab, and the cells that had penetrated to the lower surface of the membrane were fixed with $4 \%$ paraformaldehyde for $20 \mathrm{~min}$, stained with crystal violet for $5 \mathrm{~min}$ at room temperature, and counted under an inverted optical microscope (Olympus Corporation) with x100 magnification in three fields per sample.

Immunoblotting. The protein expression levels of c-Myc were determined by western blotting. A375 and A2058 cells were transfected with the miR-27b mimics, inhibitor or si-MYC for $48 \mathrm{~h}$. Protein samples were extracted from the cells using RIPA lysis buffer (Beyotime Institute of Biotechnology). The protein concentration was determined by a BCA kit (Beyotime Institute of Biotechnology). The isolated proteins $(20-100 \mu \mathrm{g})$ were separated by $10 \%$ SDS-PAGE. The proteins were transferred to polyvinylidene difluoride (PVDF) membranes (Bio-Rad 
Laboratories, Inc.). Following blocking with 3\% skim milk for $2 \mathrm{~h}$ at room temperature in Tris-buffered saline containing $0.1 \%$ Tween-20, the membranes were incubated with an anti-c-Myc primary antibody (1:1,000; cat. no. ab32072; Abcam) for $1 \mathrm{~h}$ at room temperature. Following washing with tris-buffered saline with $0.1 \%$ Tween 20 for 5 min thrice at room temperature, the membranes were incubated with HRP-conjugated secondary IgG antibodies (1:5,000; cat. no. A0208; Beyotime Institute of Biotechnology) for $1 \mathrm{~h}$ at room temperature. The signals were visualized using enhanced chemiluminescence (ECL) with a ChemiDOC XRS system (Bio-Rad Laboratories, Inc.). The densitometry analysis was performed by ImageJ 1.52 software (National Institutes of Health).

Reverse transcription-quantitative (RT-q)PCR. A375 and A2058 cells were transfected with the miR-27b mimics, inhibitor or si-MYC for $48 \mathrm{~h}$. Total RNA was extracted from the A375 and A2058 cells using TRIzol ${ }^{\circledR}$ reagent (Invitrogen; Thermo Fisher Scientific, Inc.) according to the manufacturer's instructions. Complementary DNA was synthesized from the extracted RNA using the StarScript II First-strand cDNA Synthesis Mix (cat. no. A223-2; GenStar) according to the manufacturer's protocol $\left(42^{\circ} \mathrm{C}\right.$ for $30 \mathrm{~min}$ and $85^{\circ} \mathrm{C}$ for $5 \mathrm{~min}$, followed by storage at $4^{\circ} \mathrm{C}$ ). The expression levels of miRNA and mRNA were examined using a $\mathrm{SYBR}^{\circledR}$ Green PCR Master Mix (Qiagen $\mathrm{GmbH}$ ). An ABI7500 real-time PCR detection system (Applied Biosystems; Thermo Fisher Scientific, Inc.) was used for qPCR, and the thermocycling conditions were as follows: Initial denaturation at $95^{\circ} \mathrm{C}$ for $2 \mathrm{~min}$, followed by 40 cycles of denaturation at $95^{\circ} \mathrm{C}$ for $15 \mathrm{sec}$, and annealing and extension at $60^{\circ} \mathrm{C}$ for $30 \mathrm{sec}$. GAPDH (for mRNA) or RNU6B (for miRNA) expression was used as an internal reference. The relative expression levels were calculated using the $2^{-\Delta \Delta \mathrm{Cq}}$ method (30). The primer sequences are listed in Table I.

Dual-luciferase reporter assay. The dual-luciferase reporter assay was used to determine the potential binding between miR-27b and MYC predicted by TargetScan. The wild-type vector (wt-MYC 3'-UTR) was generated by amplifying and cloning the MYC 3'-UTR into the downstream region of the Renilla psiCHECK2 vector (Promega Corporation). The mutant reporter (mut-MYC 3'-UTR) was generated by mutating the predicted miR-27b binding site in MYC 3'-UTR. Subsequently, $\sim 5 \times 10^{4} 293 \mathrm{~T}$ cells/ml (cat. no. CRL-3216; ATCC) were cultured in DMEM supplemented with 10\% FBS and were co-transfected with $1 \mu \mathrm{g} / \mathrm{ml}$ luciferase reporter vectors and $20 \mathrm{nM}$ miR-27b mimics or inhibitor using Lipofectamine ${ }^{\circledR}$ 3000 for $48 \mathrm{~h}$ at $37^{\circ} \mathrm{C}$. Following transfection, the luciferase activity was evaluated by the Dual-Luciferase Reporter Assay System (Promega Corporation) using firefly luciferase activity for normalization. The primers used for plasmid construction are listed in Table I.

RNA immunoprecipitation (RIP). The RIP assay was used to confirm the predicted binding between miR-27b and MYC 3'-UTR using a Magna RIP RNA-Binding Protein Immunoprecipitation kit (cat. no. 17-700; MilliporeSigma) according to the manufacturer's protocol. Briefly, A2058 cells were lysed using RIP lysis buffer (MilliporeSigma) and centrifuged at $10,000 \mathrm{xg}$ for $10 \mathrm{~min}$ at $4^{\circ} \mathrm{C}$ to collect the

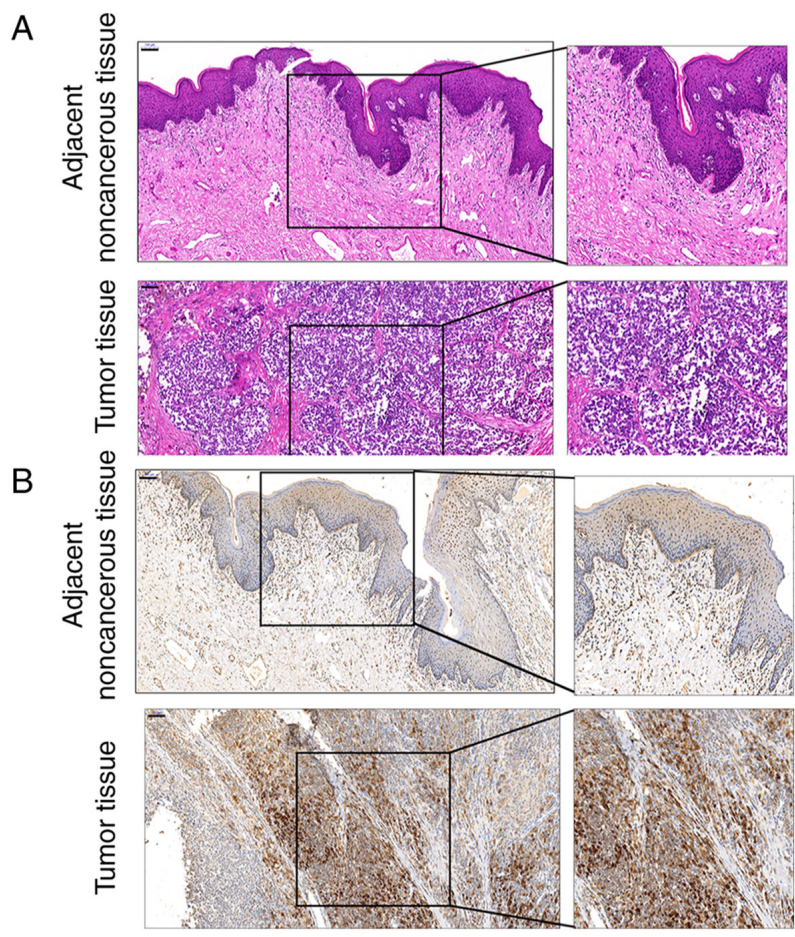

Figure 1. Protein levels and distribution of c-Myc in tissue samples. (A) Histomorphological changes in melanoma and adjacent noncancerous normal tissues were observed by hematoxylin and eosin staining (x100 magnification). (B) The protein contents and distribution of c-Myc were determined using immunohistochemical staining. c-Myc levels appeared higher in tumor tissues compared with those in adjacent noncancerous tissues (x100 magnification)

supernatant. The magnetic A/G beads $(50 \mu \mathrm{l})$ were incubated with $5 \mu$ g argonaute 2 (Ago2; cat. no. ab186733; Abcam) or IgG (cat. no. ab109489; Abcam) antibodies. Subsequently, the bead-antibody complex was rinsed, resuspended in $900 \mu \mathrm{l}$ RIP Wash Buffer, and incubated with $100 \mu \mathrm{l}$ supernatant at $4^{\circ} \mathrm{C}$ overnight. Following incubation with protease K, the RNA was extracted from the samples for RT-qPCR detection for miR-27b and the 3'UTR of MYC.

Statistical analysis. Data from triplicate experiments were analyzed with GraphPad Prism 6 software (GraphPad Software, Inc.) and presented as the mean \pm SD. Data normality was assessed by the Kolmogorov-Smirnov test. Paired Student's t-test was used for statistical comparison between tumor tissues group and adjacent noncancerous tissues group. Differences among multiple groups were determined using one-way ANOVA with Tukey's post hoc test. The correlation between miR-27b and MYC mRNA expression levels was analyzed by Pearson's correlation analysis. $\mathrm{P}<0.05$ was considered to indicate a statistically significant difference.

\section{Results}

c-Myc protein levels and distribution in melanoma and adjacent noncancerous tissue samples. To determine the mechanism of c-Myc functions in melanoma, melanoma and paired adjacent noncancerous tissue samples were collected, and the histomorphological changes in these samples were examined by H\&E staining. As demonstrated in Fig. 1A, 
A

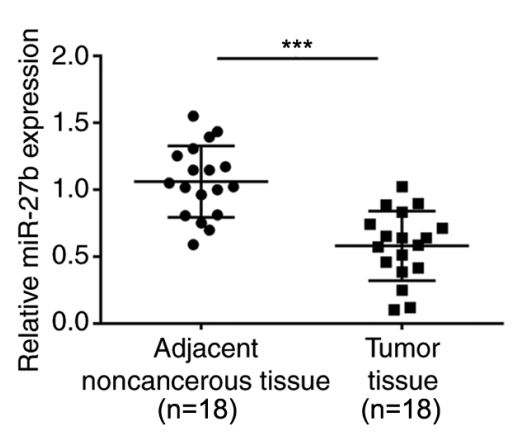

B

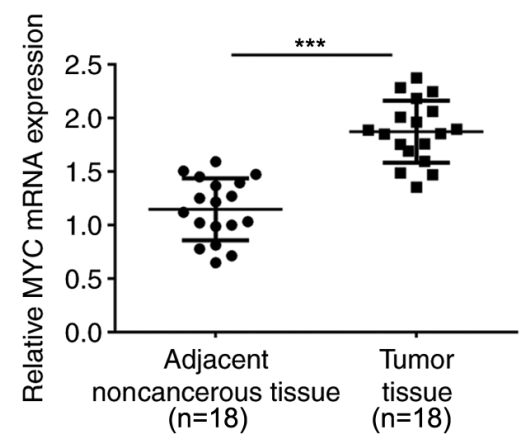

C

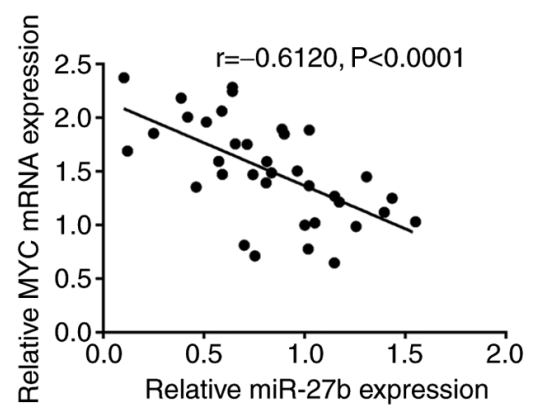

Figure 2. Expression levels and correlation of miR-27b and MYC in tissue samples from patients with melanoma. (A and B) The expression levels of miR-27b and MYC in 18 paired melanoma and adjacent noncancerous tissue samples were determined using reverse transcription-quantitative PCR. The expression levels of miR-27b were lower, whereas the MYC mRNA levels were higher in tumor tissues compared with those in the adjacent noncancerous tissues. (C) The correlation of miR-27b and MYC expression levels in patient tissue samples was analyzed using Pearson's correlation analysis. ${ }^{* * *} \mathrm{P}<0.001$. miR, microRNA.

melanoma cells were heteromorphic and exhibited no signs of maturity. IHC staining revealed that melanoma cells were c-Myc-positive (Fig. 1B).

Expression levels and correlation of miR-27b and MYC within tissues. Since the online tool TargetScan predicted that miR-27b may target MYC, the present study determined the miR-27b and MYC expression levels in 18 paired melanoma and noncancerous normal tissue samples. The expression levels of miR-27b were markedly reduced, whereas the expression levels of MYC were increased in the melanoma tissues compared with those in the adjacent noncancerous tissues (Fig. 2A and B). In addition, the expression levels of miR-27b were negatively correlated with those of MYC within the tumor and adjacent noncancerous tissues ( $r=-0.6120$; Fig. 2C), suggesting that miR-27b may target MYC to negatively regulate MYC expression levels.

Effects of miR-27b on the phenotype of melanoma cells. To validate the specific roles of miR-27b in melanoma cell lines, miR-27b expression levels were determined in a normal cell line, MC, and two melanoma cell lines, A375 and A2058, by RT-qPCR; consistent with the results observed in patient tissue samples, miR-27b expression levels were markedly lower in the two melanoma cell lines compared with those in the MCs (Fig. 3A). The melanoma cells were transfected with miR-27b mimics or inhibitor to achieve overexpress or inhibit the levels of miR-27b, and the transfection efficiency was verified by RT-qPCR; transfection with the miR-27b mimics significantly increased the cellular miR-27b levels, whereas the miR-27b inhibitor decreased the cellular miR-27b levels compared with those in the corresponding NC groups (Fig. 3B). The phenotypes of the A 375 and A 2058 cells transfected with the miR-27b mimics or inhibitor were subsequently examined. The BrdU assay results demonstrated that transfection with the miR-27b mimics significantly inhibited the DNA synthesis ability in melanoma cells compared with that in cells in the mimics-NC group (Fig. 3C). The results of the MTT and Transwell assay revealed that the A375 and A2058 cell viability and invasive ability were inhibited by the miR-27b mimics compared with those in the mimics-NC-transfected cells (Fig. 3D-G); by contrast, miR-27b inhibition promoted the DNA synthesis ability, viability and invasive ability compared with those in the cells in the inhibitor-NC group (Fig. 3D-G). Therefore, miR-27b overexpression suppressed the biological malignant behaviors of A375 and A2058 melanoma cells.

miR-27b targets MYC by binding to MYC 3'-UTR. In order to validate the binding between miR-27b and MYC predicted by the online tool TargetScan, the A375 and A2058 cell lines were transfected with the miR-27b mimics or inhibitor, and the c-Myc protein content was detected by immunoblotting. Overexpression of miR-27b downregulated the protein levels of c-Myc, whereas the inhibition of miR-27b upregulated the levels of c-Myc compared with those in the corresponding NC groups (Fig. 4A and B).

The dual-luciferase reporter assay was subsequently used. The predicted binding site is presented in Fig. 4C. The reporter vectors were co-transfected into $293 \mathrm{~T}$ cells with the miR-27b mimics or inhibitor. The luciferase activity of wt-MYC 3'-UTR was decreased by the overexpression of miR-27b, but increased following inhibition of miR-27b compared with that in the corresponding NC groups (Fig. 4D). In the presence of mut-MYC 3'-UTR, transfection with the miR-27b mimics or inhibitor did not affect luciferase activity, suggesting that miR-27b bound to the predicted binding site in the MYC 3'-UTR

To confirm the binding of miR-27b to MYC, RIP assay was performed using an Ago2 antibody. Anti-AGO2 immunoprecipitants containing miRNAs and their interacting RNA components were examined for the levels of $\mathrm{miR}-27 \mathrm{~b}$ and MYC in A2058 cells; as demonstrated in Fig. 4E, compared with the anti-IgG group, miR-27b and MYC were more abundant in the Ago2 group. In addition, the miR-27b mimics or mimics-NC were transfected into A2058 cells. Higher levels of MYC mRNA were detected in the anti-Ago2 immunoprecipitants of miR-27b mimics-transfected cells compared with those in the mimics-NC-transfected cells (Fig. 4F). These results confirmed that miR-27b bound to the MYC 3'-UTR.

miR-27b modulates the melanoma cell phenotype through $M Y C$. The present study further validated the dynamic effects of the miR-27b/MYC axis on the melanoma cell phenotype. A375 and A2058 cells were transfected with si-MYC to 
A

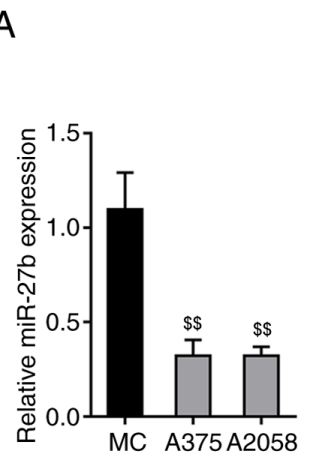

D

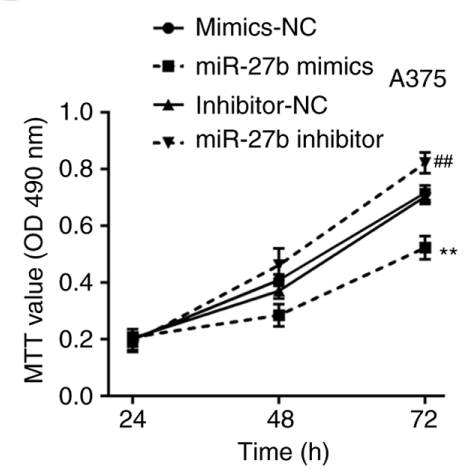

B
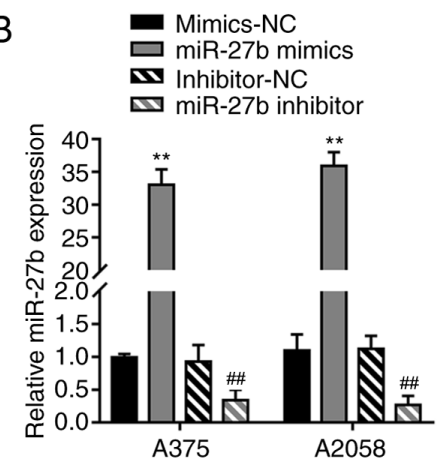

E

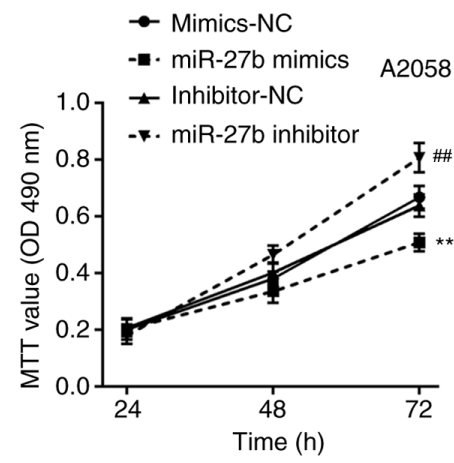

C Mimics-NC $\mathbf{W}$ Inhibitor-NC
$\square$ miR-27b mimics $\mathbf{m i R - 2 7 b}$ inhibitor

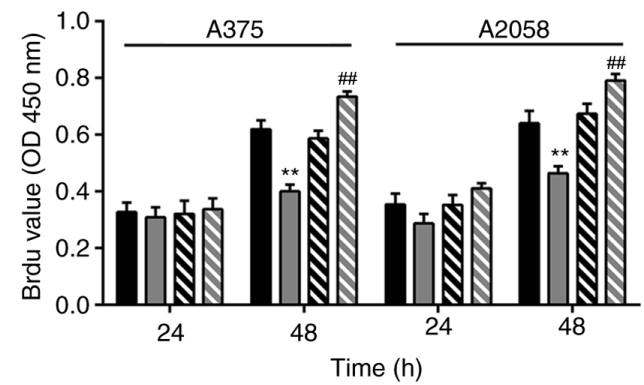

G

Mimics-NC
miR-27b mimics

F
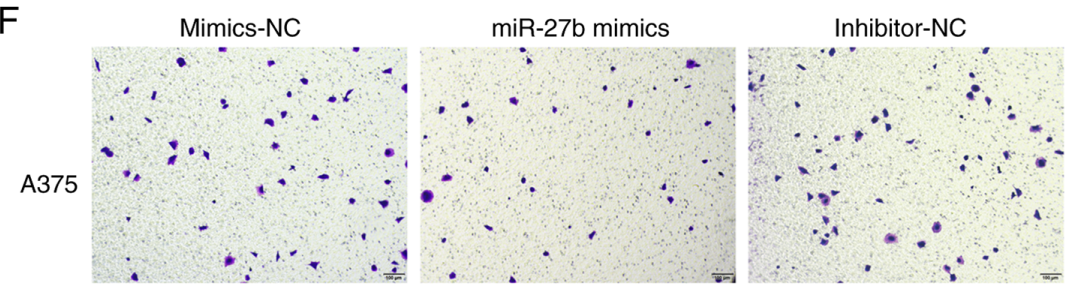

miR-27b inhibitor

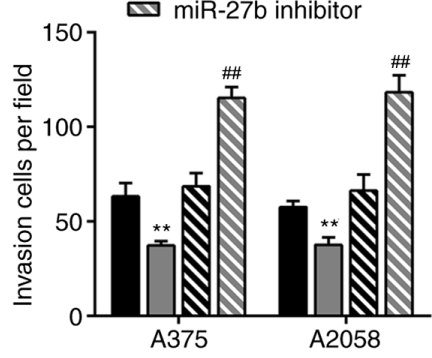

Iv Inhibitor-NC

miR-27b inhibitor

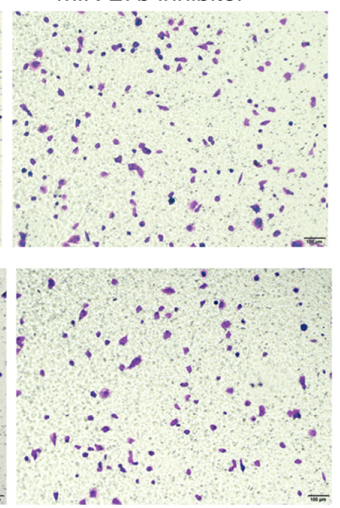

Figure 3. Effects of miR-27b on melanoma cell phenotype. (A) miR-27b expression was examined in a normal cell line, MC, and two melanoma cell lines, A375 and A2058, using RT-qPCR. miR-27b expression levels were lower in melanoma cell lines compared with those in MC. (B) miR-27b overexpression or miR-27b inhibition was achieved in two melanoma cell lines by the transfection of the miR-27b mimics or inhibitor; mimics-NC and inhibitor-NC were used as negative controls. The transfection efficiency was validated by RT-qPCR. (C) DNA synthesis ability of A375 and A2058 cells transfected with the miR-27b mimics or inhibitor by BrdU assay; the miR-27b mimics decreased, whereas the miR-27b inhibitor increased the DNA synthesis ability compared with that in the corresponding NC groups. (D and E) Cell viability was determined by MTT assay at 24, 48 and 72 h; the miR-27b mimics decreased, whereas the miR-27b inhibitor increased cell viability compared with that in the corresponding NC groups. (F and G) Cell invasive ability was determined by Transwell assay; the miR-27b mimics decreased, whereas the miR-27b inhibitor increased the invasive ability of A375 and A2058 cells compared with that in the corresponding NC groups. ${ }^{\$} \mathrm{P}<0.01$ vs. $\mathrm{MC},{ }^{* *} \mathrm{P}<0.01$ vs. mimics-NC; ${ }^{\# \#} \mathrm{P}<0.01$ vs. inhibitor-NC. miR, microRNA; MC, melanocytes; NC, negative control; BrdU, 5-bromo-2-deoxyuridine; RT-qPCR, reverse transcription-quantitative PCR.

achieve MYC knockdown; the transfection efficiency was confirmed using immunoblotting, and the results demonstrated that si-MYC successfully reduced the c-Myc protein levels compared with those in the cells transfected with si-NC (Fig. 5A and B). Subsequently, A375 and A2058 cells we co-transfected with the miR-27b inhibitor and si-MYC. The results of the in vitro assays demonstrated miR-27b inhibition promoted, whereas MYC knockdown inhibited the DNA synthesis ability (Fig. 5C), cell viability (Fig. 5D and E) and invasive ability (Fig. 5F and G) compared with those in the
si-NC and inhibitor-NC group; additionally, MYC knockdown significantly attenuated the effects of miR-27b inhibition (Fig. 5C-G). Thus, miR-27b may exert its effects on melanoma cells through MYC.

\section{Discussion}

The results of the present study demonstrated that the expression levels of miR-27b were markedly decreased in melanoma tissue samples and cells compared with those in adjacent 
A

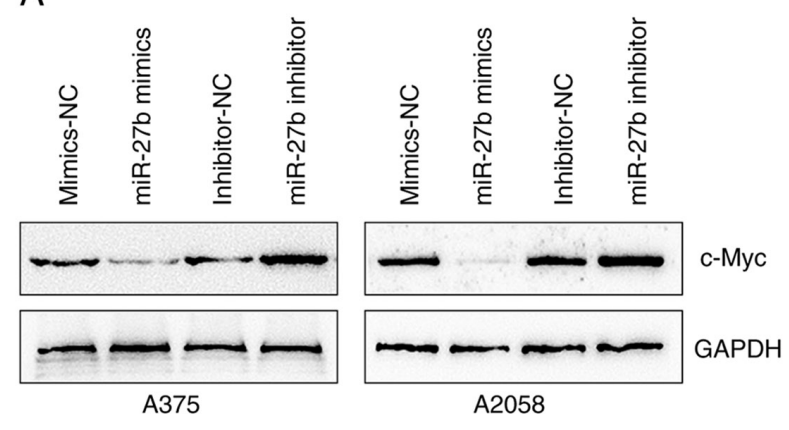

B

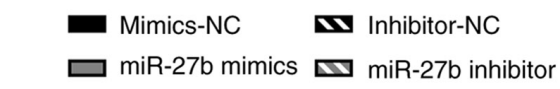

C

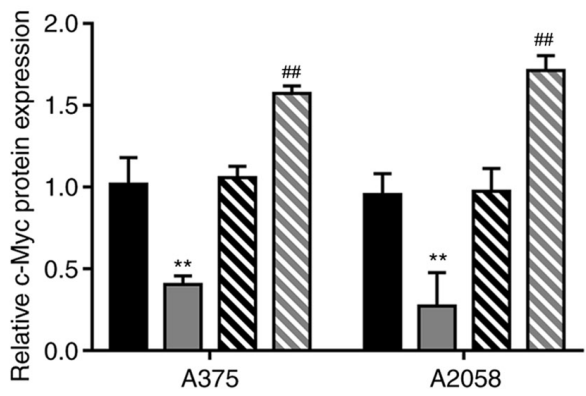

E

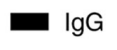

$\square \mathrm{Ago} 2$

Position 1195-1201 of MYC 3'UTR

wt-MYC 3'UTR 5'

hsa-miR-27b-5p 3'

mut-MYC 3'UTR 5'

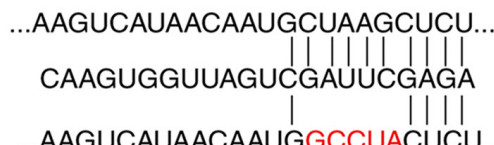

D

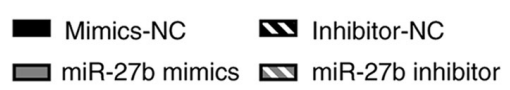

$\mathrm{F}$
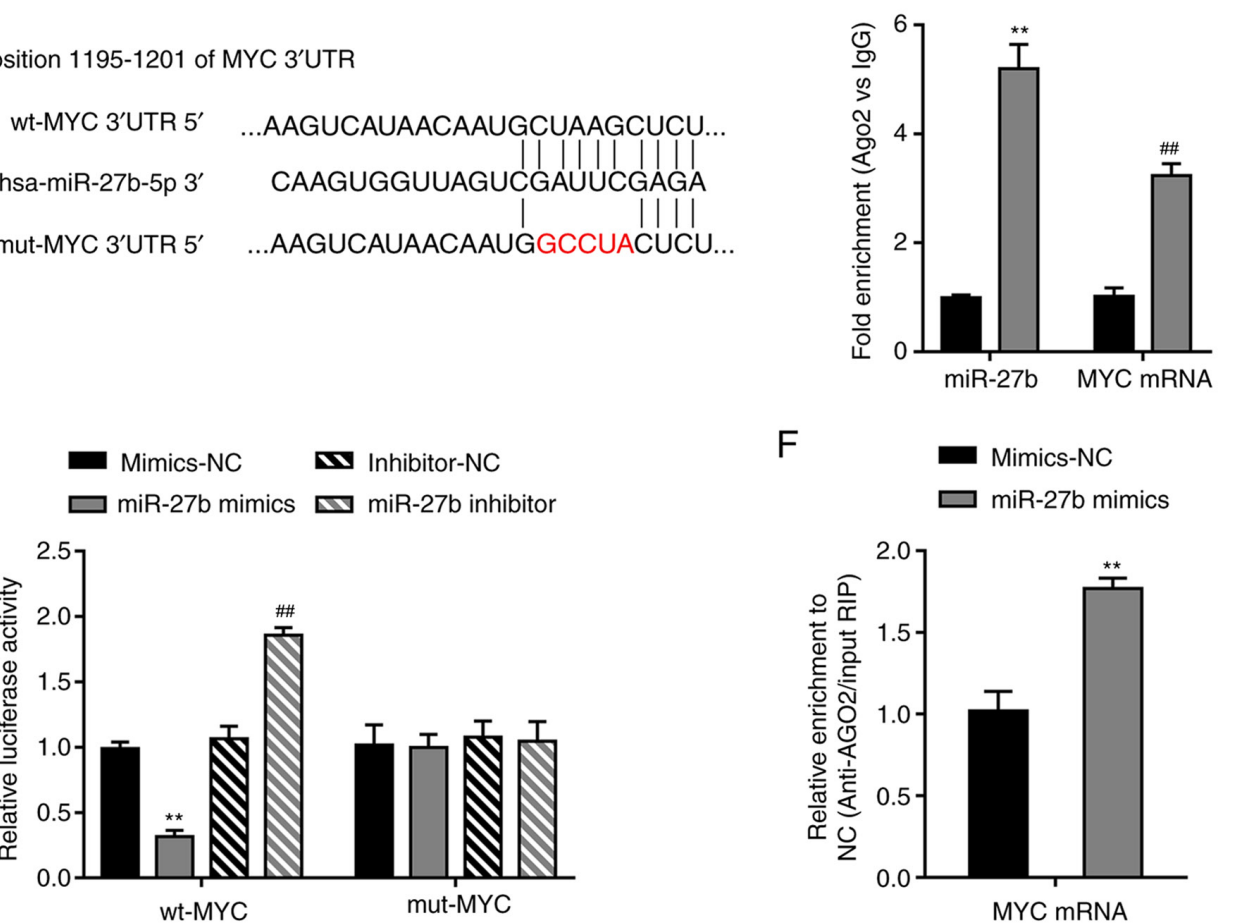
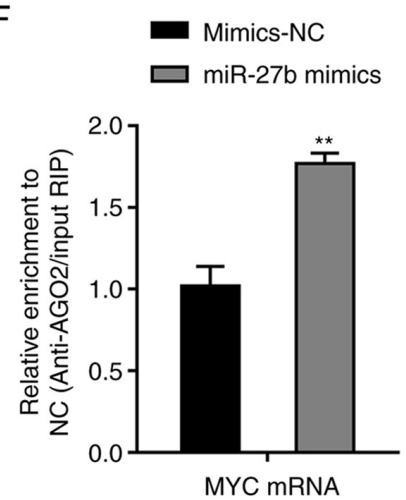

Figure 4. miR-27b targets MYC by binding to the MYC 3'-UTR. (A and B) A375 and A2058 cells were transfected with the miR-27b mimics or inhibitor, and immunoblotting revealed that the miR-27b mimics decreased, whereas the miR-27b inhibitor increased the c-Myc protein levels compared with those in the corresponding NC groups. (C) Wt and mut MYC 3'-UTR luciferase reporter vectors were constructed; the mut-MYC 3'-UTR vector contained a 5-bp mutation in the predicted miR-27b binding site. (D) Dual-luciferase reporter assay in 293T cells demonstrated that the miR-27b mimics reduced, whereas the miR-27b inhibitor increased the luciferase activity in the wt-MYC group; no changes were observed in the mut-MYC group. (E and F) RIP assay was performed to confirm the binding of miR-27b to the MYC 3'-UTR using the Ago2 antibody; the levels of miR-27b and MYC 3'-UTR in the precipitates were examined using reverse transcription-quantitative PCR. The miR-27b and MYC 3'-UTR fragments were enriched in the Ago2 precipitate compared with the IgG group. In the miR-27b mimics-transfected cells, the MYC 3'-UTR fragments were more highly enriched compared with those in the mimics-NC transfected cells. ${ }^{* *} \mathrm{P}<0.01$ vs. mimics-NC; ${ }^{\#} \mathrm{P}<0.01$ vs. inhibitor-NC. miR, microRNA; UTR, untranslated region; NC, negative control; wt, wild-type; mut, mutant; RIP, radioimmunoprecipitation; Ago2, argonaute 2.

normal tissues and normal cells, respectively. By contrast, MYC expression levels were upregulated in melanoma tissues and cells compared with those in the corresponding control groups. miR-27b overexpression significantly inhibited melanoma cell DNA synthesis ability, viability and invasive ability compared with those in cells transfected with the mimic-NC. Through binding to MYC 3'-UTR, miR-27b inhibited MYC expression. MYC knockdown in melanoma cells exerted similar effects as miR-27b overexpression on the DNA synthesis ability, cell viability and invasive ability; the effects of miR-27b inhibition were significantly reversed by MYC knockdown.
miRNAs regulate a number of biological and pathological processes, such as the biological malignant behaviors of cancer cells (23). For example, miR-21 inhibits malignant biological behaviors of melanoma (31). The abnormal regulation and dysfunction of miRNAs have been reported in patients with melanoma as well as in melanoma cells (32). In addition, miR-137, miR-148 and miR-182 have been demonstrated to regulate the levels of microphthalmia-associated transcription factor in melanoma (33). miR-26a induces apoptosis in melanoma cells by modulating SMAD1 and silencer of death domains (34). miRNAs have also been implicated in the 

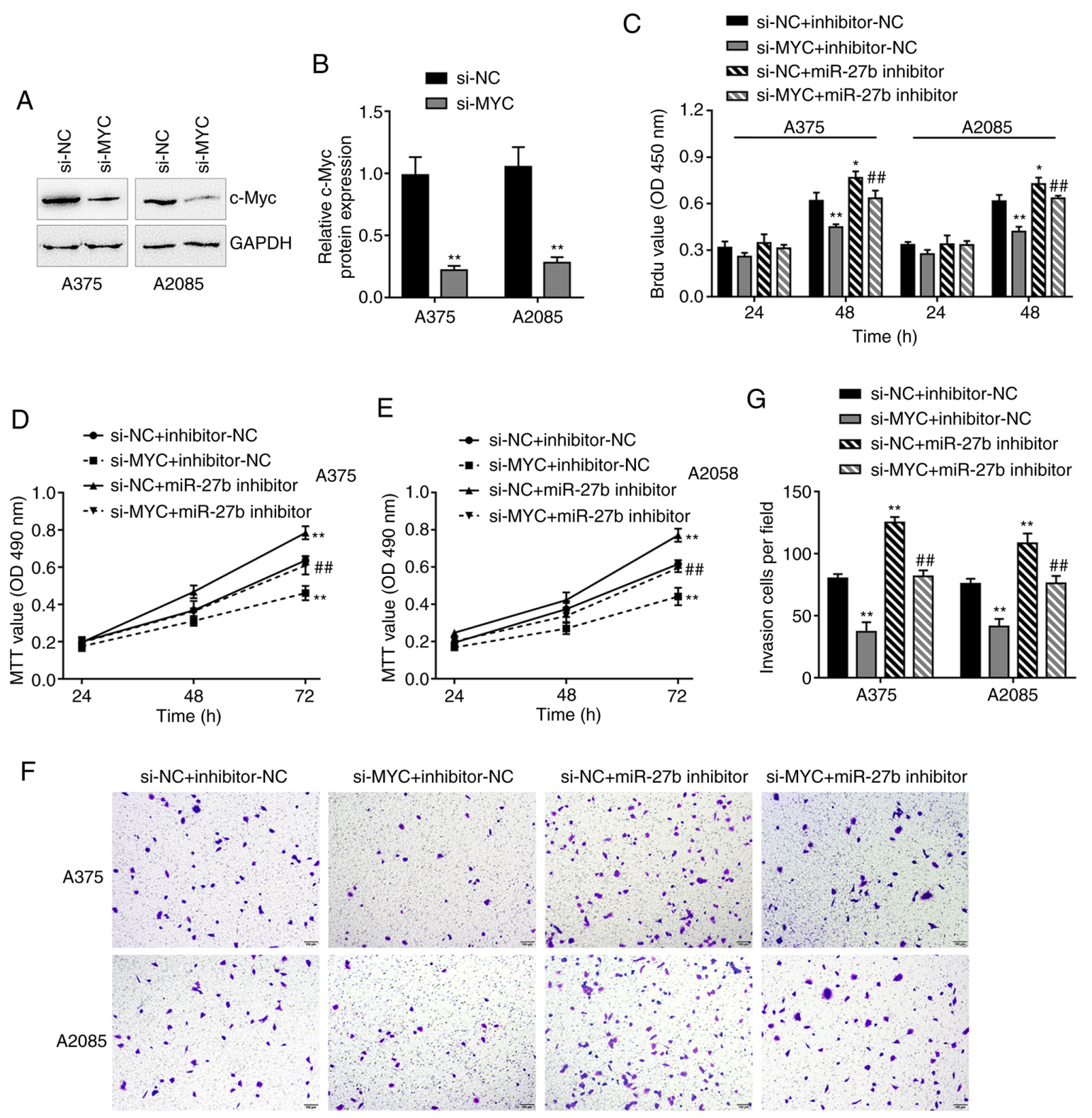

Figure 5. miR-27b modulates the melanoma cell phenotype through MYC. (A and B) MYC knockdown was achieved in A375 and A2058 cells by the transfection with si-MYC; the transfection efficiency was confirmed by western blotting. (C) A375 and A2058 cells were co-transfected with the miR-27b inhibitor and si-MYC, and the DNA synthesis ability was determined by BrdU assay; si-MYC inhibited the DNA synthesis ability compared with that in the si-NC + inhibitor-NC group, which was reversed by the miR-27b inhibitor at $48 \mathrm{~h}$ in both cell lines. (D and E) Cell viability was determined by MTT assay at 24 , 48 and $72 \mathrm{~h}$. si-MYC inhibited the cell viability compared with that in the si-NC + inhibitor-NC group, which was reversed by miR-27b inhibitor transfection. (F and G) Cell invasive ability was determined by Transwell assay. si-MYC inhibited the cell invasive ability compared with that in the si-NC + inhibitor-NC group, which was reversed by the miR-27b inhibitor. ${ }^{*} \mathrm{P}<0.05$ and ${ }^{* *} \mathrm{P}<0.01$ vs. si-NC + inhibitor-NC; \# $\mathrm{P}<0.01$ vs. si-NC + miR-27b inhibitor. miR, microRNA; si, small interfering RNA; BrdU, 5-bromo-2-deoxyuridine.

development of melanoma drug resistance and organ-specific metastasis (35). For example, a miR-150-5p, miR-15b-5p, miR-16-5p and miR-374b-3p prognostic signature distinguishes brain metastatic melanomas and non-brain metastatic primary melanomas (36). miR-200c inhibits melanoma progression and drug resistance through downregulation of BMI-1 (37). According to the online expression profiles GSE77090, a total of 58 miRNAs are downregulated in melanoma (data not shown); among them, miR-27b may target MYC, which is a proto-oncogene (17). As previously reported, miR-27b suppresses the capacity of a number of cancer cell types to proliferate, invade and migrate. For example, miR-27b inhibits the capacity of aggressive prostate cancer cells to migrate and invade, but does not affect their proliferative ability (38). By targeting peroxisome proliferator-activated receptor $\gamma$, miR-27b suppresses neuroblastoma cell proliferation, tumor development and inflammatory response (39). In colorectal cancer, miR-27b binds to vascular endothelial growth factor $\mathrm{C}$ to suppress tumor development and the formation of new blood vessels (40). In the present study, consistent with its abnormal downregulation in melanoma, miR-27b inhibition significantly promoted melanoma cell DNA synthesis ability, viability and invasive ability; by contrast, miR-27b overexpression markedly suppressed these biological malignant behaviors of melanoma cells, suggesting that miR-27b may exert a tumor-suppressive effect on melanoma. 
Regarding the mechanisms by which miRNAs exert their biological functions, miRNAs are considered to target various downstream mRNAs, leading to translation inhibition, mRNA deadenylation or decay (21). Based on the TargetScan analysis performed in the preliminary experiments of the present study, it was determined that miR-27b may target the 3'-UTR of MYC, which was confirmed by experimental analyses. The c-Myc proto-oncogene is located on chromosome 8 , and a variety of anomalies related to its activated expression occur during the development of numerous types of malignant tumors (41). c-Myc is considered to modulate the expression of $\leq 15 \%$ of all human genes (16). A number of genes modulated by c-Myc are involved in cell proliferation, migration, invasion and apoptosis, thus promoting the occurrence and development of tumors (42-45). A previous study supports the suggestion that oncogenic c-Myc affects the presence of determinants of immunological importance on melanoma cells (46). c-Myc overexpression promotes melanoma metastasis by promoting vasculogenic mimicry (18). By contrast, MYC depletion results in the repression of the expression of a number of genes encoding enzymes that are rate-limiting for dNTP metabolism, including phosphoribosyl pyrophosphate synthetase 2 and inosine monophosphate dehydrogenase 2 , resulting in the inhibition of melanoma proliferation (47). In the present study, MYC mRNA expression levels were demonstrated to be upregulated in melanoma tissue samples compared with those in paired adjacent noncancerous tissues. MYC knockdown in melanoma cells significantly inhibited the DNA synthesis ability, viability and invasive ability compared with those in the cells transfected with si-NC. In addition, miR-27b served as a tumor suppressor in melanoma cells, as miR-27b overexpression inhibited melanoma cell proliferation and invasive capability compared with those in the NC group. By contrast, miR-27b inhibition promoted cell proliferation and invasion compared with those in the inhibitor-NC-transfected cells. Additionally, MYC expression levels were reduced by miR-27b overexpression and increased by miR-27b inhibition compared with those in the corresponding NC groups. MYC knockdown significantly reversed the effects of miR-27b inhibition, indicating that miR-27b may serve as a tumor suppressor in melanoma by targeting MYC. Due to the crucial role of c-Myc in melanoma progression, targeting c-Myc is considered a potential therapeutic strategy for melanoma. In a previous study, nanodelivery of the c-Myc inhibitor 10058-F4 effectively inhibited human and mouse melanoma cell proliferation (10). Nanoparticle-delivered si-c-Myc sensitizes melanoma cells to paclitaxel and inhibits tumor growth (48).

In conclusion, the results of the present study demonstrated that the miR-27b/MYC axis modulated melanoma cell biological malignant behaviors, suggesting that it may be a potential target for melanoma treatment.

\section{Acknowledgements}

Not applicable.

\section{Funding}

This study was supported by the National Natural Science Foundation of China (grant no. 81774325) and the project of the Hunan Province Health Committee (grant no. 20201683).

\section{Availability of data and materials}

All data generated or analyzed during this study are included in this published article.

\section{Authors' contributions}

YT and JZ performed the experiments and wrote the manuscript. ZY supervised the research. JZ revised the manuscript. ZY designed the study. YT, ZY and JZ confirm the authenticity of all the raw data. All authors read and approve the final manuscript.

\section{Ethics approval and consent to participate}

The study was approved by the Ethics Committee of the Second Affiliated Hospital of Hunan University of Chinese Medicine (approval No. 2019-KY-031; Changsha, China). All patients provided written informed consent.

\section{Patient consent for publication}

Not applicable.

\section{Competing interests}

The authors declare that they have no competing interests.

\section{References}

1. Dimitriou F, Krattinger R, Ramelyte E, Barysch MJ, Micaletto S, Dummer R and Goldinger SM: The world of melanoma: Epidemiologic, genetic, and anatomic differences of melanoma across the globe. Curr Oncol Rep 20: 87, 2018.

2. Carr S, Smith C and Wernberg J: Epidemiology and risk factors of melanoma. Surg Clin North Am 100: 1-12, 2020.

3. Schadendorf D, van Akkooi ACJ, Berking C, Griewank KG, Gutzmer R, Hauschild A, Stang A, Roesch A and Ugurel S: Melanoma. Lancet 392: 971-984, 2018.

4. Lopez-Soto A, Gonzalez S, Smyth MJ and Galluzzi L: Control of metastasis by NK cells. Cancer Cell 32: 135-154, 2017.

5. Bhandaru M and Rotte A: Monoclonal antibodies for the treatment of melanoma: Present and future strategies. Methods Mol Biol 1904: 83-108, 2019.

6. McDermott D, Haanen J, Chen TT, Lorigan P and O'Day S; MDX010-20 investigators: Efficacy and safety of ipilimumab in metastatic melanoma patients surviving more than 2 years following treatment in a phase III trial (MDX010-20). Ann Oncol 24: 2694-2698, 2013.

7. Weiss SA, Wolchok JD and Sznol M: Immunotherapy of melanoma: Facts and hopes. Clin Cancer Res 25: 5191-5201, 2019.

8. Paluncic J, Kovacevic Z, Jansson PJ, Kalinowski D, Merlot AM, Huang MLH, Lok HC, Sahni S, Lane DJR and Richardson DR: Roads to melanoma: Key pathways and emerging players in melanoma progression and oncogenic signaling. Biochim Biophys Acta 1863: 770-784, 2016.

9. Ankeny JS, Labadie B, Luke J, Hsueh E, Messina J and Zager JS: Review of diagnostic, prognostic, and predictive biomarkers in melanoma. Clin Exp Metastasis 35: 487-493, 2018.

10. Karagiannis P, Fittall $M$ and Karagiannis SN: Evaluating biomarkers in melanoma. Front Oncol 4: 383, 2015.

11. Sun J, Zhang D, Bae DH, Sahni S, Jansson P, Zheng Y, Zhao Q, Yue F, Zheng M, Kovacevic Z and Richardson DR: Metastasis suppressor, NDRG1, mediates its activity through signaling pathways and molecular motors. Carcinogenesis 34: 1943-1954, 2013.

12. Smalley MJ and Dale TC: Wnt signalling in mammalian development and cancer. Cancer Metastasis Rev 18: 215-230, 1999

13. Zheng $L$ and Pan J: The anti-malarial drug artesunate blocks wnt/beta-catenin pathway and inhibits growth, migration and invasion of uveal melanoma cells. Curr Cancer Drug Targets 18: 988-998, 2018. 
14. Sinnberg T, Levesque MP, Krochmann J, Cheng PF, Ikenberg K, Meraz-Torres F, Niessner $\mathrm{H}$, Garbe $\mathrm{C}$ and Busch C: Wnt-signaling enhances neural crest migration of melanoma cells and induces an invasive phenotype. Mol Cancer 17: 59, 2018.

15. Clevers H: Wnt/beta-catenin signaling in development and disease. Cell 127: 469-480, 2006.

16. Gearhart J, Pashos EE and Prasad MK: Pluripotency redux-advances in stem-cell research. N Engl J Med 357: 1469-1472, 2007.

17. Meškyte EM, Keskas S and Ciribilli Y: MYC as a multifaceted regulator of tumor microenvironment leading to metastasis. Int J Mol Sci 21: 7710, 2020.

18. Lin X, Sun R, Zhao X, Zhu D, Zhao X, Gu Q, Dong X, Zhang D, Zhang Y, Li Y and Sun B: C-myc overexpression drives melanoma metastasis by promoting vasculogenic mimicry via c-myc/snail/Bax signaling. J Mol Med (Berl) 95: 53-67, 2017.

19. Calin GA and Croce CM: MicroRNA signatures in human cancers. Nat Rev Cancer 6: 857-866, 2006.

20. Nishikawa S, Ishii H, Haraguchi N, Kano Y, Fukusumi T, Ohta K, Ozaki M, Dewi DL, Sakai D, Satoh T, et al: microRNA-based cancer cell reprogramming technology. Exp Ther Med 4: 8-14, 2012.

21. Ha M and Kim VN: Regulation of microRNA biogenesis. Nat Rev Mol Cell Biol 15: 509-524, 2014.

22. Noori J, Sharifi M and Haghjooy Javanmard S: miR-30a inhibits melanoma tumor metastasis by targeting the E-cadherin and zinc finger E-box binding homeobox 2. Adv Biomed Res 7: 143, 2018

23. Pencheva N, Tran H, Buss C, Huh D, Drobnjak M, Busam K and Tavazoie SF: Convergent multi-miRNA targeting of ApoE drives LRP1/LRP8-dependent melanoma metastasis and angiogenesis. Cell 151: 1068-1082, 2012.

24. Gajos-Michniewicz A and Czyz M: Role of miRNAs in melanoma metastasis. Cancers (Basel) 11: 326, 2019.

25. Sole C, Tramonti D, Schramm M, Goicoechea I, Armesto M, Hernandez LI, Manterola L, Fernandez-Mercado M, Mujika K, Tuneu A, et al: The circulating transcriptome as a source of biomarkers for melanoma. Cancers (Basel) 11: 70, 2019.

26. Wang X, Zou M, Li J, Wang B, Zhang Q, Liu F and Lü G: LncRNA H19 targets miR-22 to modulate H2O2-induced deregulation in nucleus pulposus cell senescence, proliferation, and ECM synthesis through Wnt signaling. J Cell Biochem 119: 4990-5002, 2018.

27. Agarwal V, Bell GW, Nam JW and Bartel DP: Predicting effective microRNA target sites in mammalian mRNAs. ELife 4 e05005, 2015.

28. Ritchie ME, Phipson B, Wu D, Hu Y, Law CW, Shi W and Smyth GK: limma powers differential expression analyses for RNA-sequencing and microarray studies. Nucleic Acids Res 43: e47, 2015.

29. Team RC: R: A language and environment for statistical computing. R Foundation for Statistical Computing, Vienna, 2013

30. Livak KJ and Schmittgen TD: Analysis of relative gene expression data using real-time quantitative PCR and the 2(-Delta Delta C(T)) method. Methods 25: 402-408, 2001.

31. Javanmard SH, Vaseghi G, Ghasemi A, Rafiee L, Ferns GA, Esfahani HN and Nedaeinia R: Therapeutic inhibition of microRNA-21 (miR-21) using locked-nucleic acid (LNA)-anti-miR and its effects on the biological behaviors of melanoma cancer cells in preclinical studies. Cancer Cell Int 20: $384,2020$.

32. Lorusso C, De Summa S, Pinto R, Danza K and Tommasi S: miRNAs as key players in the management of cutaneous melanoma. Cells 9: 415, 2020
33. Kunz M: MicroRNAs in melanoma biology. Adv Exp Med Biol 774: 103-120, 2013

34. Qian H, Yang C and Yang Y: MicroRNA-26a inhibits the growth and invasiveness of malignant melanoma and directly targets on MITF gene. Cell Death Discov 3: 17028, 2017.

35. Ryu B, Hwang S and Alani RM: MicroRNAs as an emerging target for melanoma therapy. J Invest Dermatol 133: 1137-1139, 2013.

36. Hanniford D, Zhong J, Koetz L, Gaziel-Sovran A, Lackaye DJ, Shang S, Pavlick A, Shapiro R, Berman R, Darvishian F, et al: A miRNA-based signature detected in primary melanoma tissue predicts development of brain metastasis. Clin Cancer Res 21: 4903-4912, 2015.

37. Liu S, Tetzlaff MT, Cui R and Xu X: miR-200c inhibits melanoma progression and drug resistance through down-regulation of BMI-1. Am J Pathol 181: 1823-1835, 2012.

38. Ishteiwy RA, Ward TM, Dykxhoorn DM and Burnstein KL: The microRNA-23b/-27b cluster suppresses the metastatic phenotype of castration-resistant prostate cancer cells. PLoS One 7: e52106, 2012.

39. Lee JJ, Drakaki A, Iliopoulos D and Struhl K: MiR-27b targets PPARg to inhibit growth, tumor progression and the inflammatory response in neuroblastoma cells. Oncogene 31: 3818-3825, 2012.

40. Ye J, Wu X, Wu D, Wu P, Ni C, Zhang Z, Chen Z, Qiu F, Xu J and Huang J: miRNA-27b targets vascular endothelial growth factor $\mathrm{C}$ to inhibit tumor progression and angiogenesis in colorectal cancer. PLoS One 8: e60687, 2013.

41. Stine ZE, Walton ZE, Altman BJ, Hsieh AL and Dang CV: MYC, metabolism, and cancer. Cancer Discov 5: 1024-1039, 2015.

42. Campbell KJ and White RJ: MYC regulation of cell growth through control of transcription by RNA polymerases I and III. Cold Spring Harb Perspect Med 4: a018408, 2014.

43. Zhang Q, Spears E, Boone DN, Li Z, Gregory MA and Hann SR: Domain-specific c-Myc ubiquitylation controls c-Myc transcriptional and apoptotic activity. Proc Natl Acad Sci USA 110: 978-983, 2013

44. Kong LM, Liao CG, Zhang Y, Xu J, Li Y, Huang W, Zhang Y, Bian $\mathrm{H}$ and Chen $\mathrm{ZN}$ : A regulatory loop involving miR-22, Sp1, and c-Myc modulates CD147 expression in breast cancer invasion and metastasis. Cancer Res 74: 3764-3778, 2014.

45. Zhang L, Hou Y, Ashktorab H, Gao L, Xu Y, Wu K, Zhai J and Zhang L: The impact of C-MYC gene expression on gastric cancer cell. Mol Cell Biochem 344: 125-135, 2010.

46. Poźniak J, Nsengimana J, Laye JP, O'Shea SJ, Diaz JMS, Droop AP, Filia A, Harland M, Davies JR, Mell T, et al: Genetic and environmental determinants of immune response to cutaneous melanoma. Cancer Res 79: 2684-2696, 2019.

47. Mannava S, Grachtchouk V, Wheeler LJ, Im M, Zhuang D, Slavina EG, Mathews CK, Shewach DS and Nikiforov MA: Direct role of nucleotide metabolism in C-MYC-dependent proliferation of melanoma cells. Cell Cycle 7: 2392-2400, 2008.

48. Chen Y, Bathula SR, Yang Q and Huang L: Targeted nanoparticles deliver siRNA to melanoma. J Invest Dermatol 130: 2790-2798, 2010 .

This work is licensed under a Creative Commons Attribution-NonCommercial-NoDerivatives 4.0 International (CC BY-NC-ND 4.0) License. 